Published in final edited form as:

Pediatrics. 2008 April ; 121(4): 831-832. doi:10.1542/peds.2008-0068.

\title{
First, Do No Harm
}

\author{
Daniel K. Benjamin Jr \\ Department of Pediatrics, Duke University, Duke Clinical Research Institute, Durham, NC
}

Healy and colleagues ${ }^{1}$ are to be commended for their continued study of fluconazole resistance, one of the potential negative effects of systemic antifungal prophylaxis. The authors provide modest new evidence to support fluconazole use, provided that we first correctly investigate the risks and benefits of fluconazole prophylaxis.

All drugs cause harm to at least a small proportion of children who receive them. Some drugs reduce or cure disease in children, and a very few drugs benefit more children than are harmed by their use. The pressing public health concern regarding the adoption of fluconazole prophylaxis hinges on this risk-benefit ratio. Although antifungal prophylaxis has reduced candidiasis in the high-incidence setting, investigators have not answered:

1. Does prophylaxis reduce neonatal mortality,

2. Is prophylaxis beneficial in nurseries with low-moderate incidence of candidiasis,

3. What are the effects of prophylaxis on subsequent neurodevelopment?

\section{All cause mortality}

Mortality and candidiasis are competing outcomes and although incorporating competing outcomes into trial design seems obvious (infants who die at age 12 days cannot develop candidiasis at age 15 days), it is often ignored. The composite endpoint of death and invasive candidiasis is virtually identical in pre-prophylaxis era compared to the prophylaxis era in Healy's 2005 study $^{2}$. Prevention should either document improved mortality, or improvement in a composite endpoint that includes mortality (e.g., death or candidiasis), or improvement in a validated measure of morbidity relevant to function (e.g., neurodevelopmental outcome). No trial completed to date has documented fluconazole prophylaxis results in any one of these three areas.

\section{Benefit to the mainstream}

The concerns of long-term risk-benefit are crucial to the majority of us who work in nurseries where the incidence of invasive candidiasis in neonates born $750-1000 \mathrm{~g}$ is $1-4 \%$ because we must expose so many infants to help so few. Unfortunately, large differences in neonatal infection rates by center have resulted in a 'prophylaxis paradox'; specifically, prophylaxis studies have been conducted in a few nurseries with extremely high incidence of disease, those with infection rates 3-10 fold higher than the national median ${ }^{3,4}$. The observed reductions in positive cultures have led several authors to conclude that those of us in the mainstream (lower incidence settings) should adopt widespread use. Those of us with low incidence of disease (due to effective infection control, antimicrobial stewardship, or other factors), are wise to be less than enthusiastic because we must expose 100 infants to prophylaxis for every prevented case.

The prophylaxis paradox (not unique to invasive fungal infections) is the phenomenon that although prophylaxis may be effective where it is studied (the high incidence setting), it should not necessarily be adopted by all (low and moderate incidence) units. The cause of the prophylaxis paradox is an example of sample selection bias: sites that participate and often 
lead enrollment in prophylaxis studies are the high incidence sites that naturally view the infection under study as a public health threat. Resolution of the paradox is the conduct of simple trials with a large number of centers whose median incidence of disease reflects the broader community.

\section{Neurodevelopmental consequences}

Healy et al. correctly note that most infants $<1000 \mathrm{~g}$ birth weight who develop candidiasis suffer death or disability ${ }^{3}$; however, no group has provided even a modest amount of neurodevelopmental follow up after prophylaxis.

Neurodevelopmental follow up is the key question that must be answered prior to routinely exposing tens of thousands of extremely premature infants with a rapidly developing brain to a pharmacologic agent for 6 weeks every year for the foreseeable future. This should be answered in the form of a well-powered blinded trial that compares neurologic outcomes between exposed and un-exposed infants prior to advocating widespread adoption of fluconazole prophylaxis.

\section{References}

1. Healy CM, Campbell JR, Zaccaria E, Baker CJ. Fluconazole Prophylaxis in Extremely Low Birth Weight Neonates Reduces Invasive Candidiasis Mortality Without Emergence of FluconazoleResistant Candida Species. Pediatrics. 2008

2. Cotten CM, McDonald S, Stoll B, Goldberg RN, Poole K, Benjamin DK Jr. The Association of Third Generation Cephalosporin Use and Invasive Candidiasis in Extremely Low Birth Weight Infants. Pediatrics 2006;118:717-722. [PubMed: 16882828]

3. Benjamin DK Jr, Stoll BJ, Fanaroff AA, McDonald SA, Oh W, Higgins R, Duara S, Poole K, Laptook A, Goldberg R. Neonatal Candidiasis Among Extremely Low Birth Weight Infants: Risk Factors, Mortality, and Neurodevelopmental Outcomes at 18-22 Months. Pediatrics 2006;117:84-92. [PubMed: 16396864]

4. Healy CM, Baker CJ, Zaccaria E, Campbell JR. Impact of fluconazole prophylaxis on incidence and outcome of invasive candidiasis in a neonatal intensive care unit. J Pediatr 2005;147:166-171. [PubMed: 16126043] 